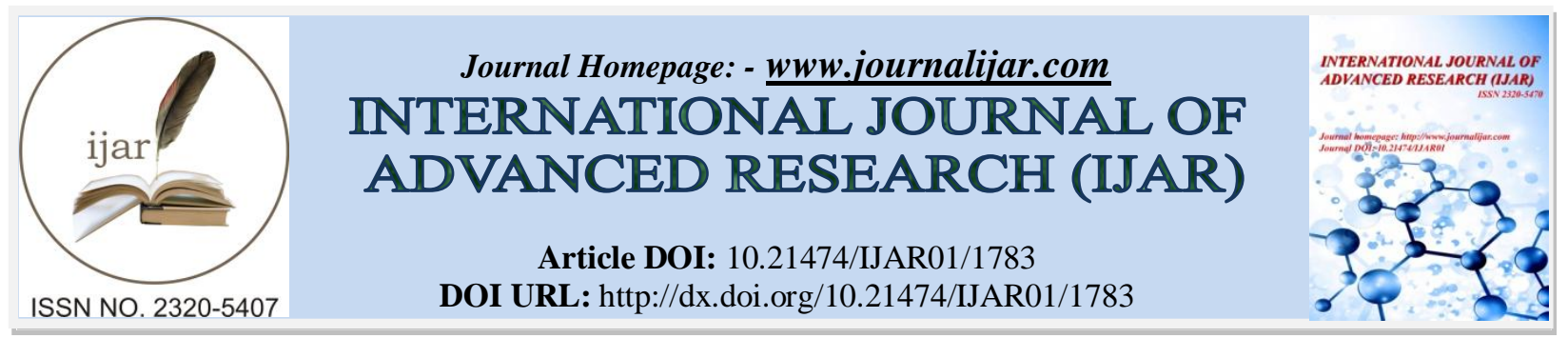

RESEARCH ARTICLE

\title{
MUTATIONAL ANALYSIS OF EXON 10 AND EXON 13 OF ATP7B GENE IN PATIENTS WITH WILSON'S DISEASE.
}

Haidar A. Shamran ${ }^{1}$, Ahdaf A. Jameel ${ }^{2}$ and Subah J. Hamza ${ }^{2}$.

1. Medical research Unit, School of Medicine, University of AL-Nahrain, Baghdad- Iraq.

2. Biotechnology Department, School of Science, University of Baghdad.

\section{Manuscript Info}

Manuscript History

Received: 12 August 2016

Final Accepted: 22 September 2016

Published: October 2016

\section{Abstract}

Background:- Wilson disease (WD) is a rare inherited autosomal recessive disorder of copper metabolism due to mutations in a copper transporter gene (ATP7B), resulting in hepatic and neuropsychiatric manifestations. It is very difficult to rely upon clinical and traditional laboratory findings for diagnosis especially in the early stages of the disease.

Aims:- To determine the most common mutations in the exon 10 and exon 13 of ATP7B gene in Iraqi patients with WD.

Subjects and Methods:- A total of 35 patients with WD and 10 apparently healthy controls were recruited for this study. Whole blood samples were collected from each subject. DNA was isolated for whole blood and the exon 10 and 13 were amplified with specific primers using PCR. PCR products were directly sequenced and the results were aligned to the published human genomic database using BLAST function. Serum samples were used for traditional laboratory investigations.

Results:- Seven different mutations have been recorded. These includes three nucleotide polymorphisms (SNPs):Lys832Arg, Pro840Leu and Thr991Thr with 22.86\%, 25.71\% and $4.29 \%$ frequencies respectively; two point mutations: Ala1003Valand Lys1010Arg which had $8.57 \%$ and $11.42 \%$ frequencies respectively and two fame-shift mutations: c.2977-2978insA and c.2457delA with frequency of $24.29 \%$ and $27.14 \%$ respectively among WD patients.

Conclusions:- These data strongly indicate that both c.2519C $>$ T polymorphism and the frame-shift mutation c.2977-2978insA could be exploited in the development of molecular diagnosis of WD.Our research has enriched the mutation spectrum of the ATP7B gene in the Iraqi population and can serve as the basis for genetic counseling and clinical/prenatal diagnosis to prevent WD in iraq. However, further studies are required to find out the most prevalent mutations in other exons.

Copy Right, IJAR, 2016,. All rights reserved. 


\section{Introduction:-}

Wilson disease (WD) also called hepatolenticular degeneration is a rare inherited autosomal recessive disorder of copper metabolism due to mutations a copper transporter gene (ATP7B), resulting in hepatic and neuropsychiatric manifestations due to copper accumulation (Mathur et al. 2015; Chen et al. 2015). Although it has been recognized and described for nearly a century, it still far from completely understood.

Wilson disease is a relatively rare disease, found worldwide, Although reliable data on the prevalence of the disease is scarce, it is estimated to be 1 case in 30,000 live births in some populations to $1 / 100000$ in most populations with carrier frequency of 1 in 90 to 122, however, the prevalence of WD has been re-evaluated in different clinical studies (Cocoş et al. 2014; Li et al. 2011). Wilson's disease may present at any age, nonetheless, the majority of cases presents between ages 5 and 35 years (European Association for the Study of liver 2012).

The underlying molecular mechanisms for WD have been extensively studied. It is now believed that a defect in the copper-transporting P-type adenosine triphosphatase (ATPase) (Wu et al. 2015) lead to impairment in the processes of incorporation of copper into ceruloplasmin and excretion of excess copper into bile. This defect occur secondary to one of several mutations in the ATP7B gene (Schilsky, 2007). The genetic linkage studies narrowed the assignment of the Wilson disease locus to 13q14-q21 (Javed et al. 2008; Dong \& Wu 2012; Kodama et al. 2012; Mathur et al. 2015). Furthermore, molecular genetic analysis reveals at least 300 distinct mutations. While most reported mutations occur in single families, only few mutations are more common. However, there is a wide range of mutations and the frequency of each of them varies considerably from country to country (Ferenci 2006).

Worldwide there is a dramatic improvement of analytic tools and the genetic testing became an integral part for the diagnosis of WD (Ferenci 2006). The identification of distribution of particular mutations will help to design shortcuts for genetic diagnosis of WD. In Iraq, there are very few studies concerning the mutational profile of ATP7B gene (Al-Mayahi et al., 2016). Therefore the current study was designed to determine the most common mutations in the exon 10 and exon 13 of ATP7B gene in Iraqi patients with WD which can open a new era for the possibility for molecular diagnosis of Wilson disease.

\section{Study subjects:-}

Patients attending hospitals to undergo periodic checks to follow progress their condition with WD from January 2014 to January 2015 were eligible for this study. Two hospitals in Iraq were included: Baghdad Medical City Digestive Diseases Hospital andAL-Kadhimiyia Teaching hospital. Ethical clearance to conduct the research was sought and obtained from these hospitals. Selection of patients was accomplished with assistance of physician.

Thirty-five patients were selected to be investigated in this study. All had WD of different stages. Data were collected through direct interview with the patient, and by seeking his/her hospital record as well as previous medical reports. Ten age-matched apparently healthyindividuals were selected from students of college of Medicine/ Al-Nahrain University to represent control group. Informed consent from patients as well as control was taken which included age, sex, dwelling, and first relative family history of WD.

\section{Blood Samples:-}

Five- milliliter of blood was taken from patients and controls. Each sample was divided into two parts, $2 \mathrm{ml}$ of which was kept in EDTA tube ( used for DNA extraction and kept $-20^{\circ} \mathrm{C}$ ) and the other $3 \mathrm{ml}$ was put in plan tube and which underwent centrifugation where the serum was obtained and preserved at $-20^{\circ} \mathrm{C}$ until be used .

\section{DNA Extraction and Genotyping:-}

DNA was extracted from blood samples using ready kit ( gSYNCTM DNA Mini Kit Whole Blood Protocol / Geneaid / Korea ) procedure was done according to the manufacturer's instructions. Two pairs of primers were used for amplification of exon 10 and exon 13 of ATP7B gene. The forward and reverse primers for exon 10 were 5' GTGACCGAATGAGTGGC - 3'and 3' - TTTCCCAGAACTCTTCACA -5' respectively, while those for exon 13 were 5'-GAAATGTCCTTATGTGATT-3'and 3'- AGTAAACAGATACTACTTTCATC - 5' respectively. Template DNA $(10 \mu \mathrm{l})$ fromsample and primers $(5 \mu \mathrm{l})$ were added to master-mix tube. The mixture put in shaker and spinner 10 times for better mixing. After mixing, the mastermix tubes were transferred to the thermocycler (MyGenie 32 thermal block / Bioneer/ Korea) which is previously programmed with the above protocol according to gene to be amplified. The PCR conditions for both exons included an initial denaturation for $5 \mathrm{~min}$ at $95{ }^{\circ} \mathrm{C}$ followed 
by 35 cycles of denaturation for $30 \mathrm{sec}$ at $94^{\circ} \mathrm{C}$, annealing for $30 \mathrm{sec}$ at $65^{\circ} \mathrm{C}$ and an elongation for $1 \mathrm{~min}$ at $72 \mathrm{C}^{\circ} \mathrm{A}$ final elongation for $10 \mathrm{~min}$ at $72^{\mathrm{C}} \mathrm{C}$ was applied as a final step. PCR products were sent for Macrogen/ Korea for direct sequencing. The sequenced DNA was edited using Chromas Pro v1.5 (Technelysium Pty Ltd) and aligned to the published human genomic database using BLAST function from pubmed. DNA mutation numbering was based on c.DNA.

\section{Results:-}

Table 1:- shows demographic and clinical features of WD patients.

Table 1:- Demographic and clinical features of WD patients.

\begin{tabular}{|l|l|}
\hline Index & Value (mean \pm SD) \\
\hline Age (mean \pm SD) & \\
\hline Sex (Male: female) & $20: 15$ \\
\hline Residence (urban: rural) & $24: 11$ \\
\hline parental consanguinity (Consanguineous/ non-cons.) & $14: 21$ \\
\hline ALT (IU/L) & $132.14 \pm 23.12$ \\
\hline AST (IU/L) & $96.93 \pm 11.21$ \\
\hline ALP(IU/L) & $184.29 \pm 48.45$ \\
\hline Serum bilirubin(IU/L) & $3.6 \pm 1.13$ \\
\hline Serum ceruloplasmin & $30.3 \pm 14.39(\mathrm{mg} / \mathrm{dl})$ \\
\hline Serum copper $(\mu \mathrm{g} / \mathrm{dl})$ & $127.18 \pm 64.432$ \\
\hline Urinary copper / 24 h & $118.12 \pm 23.86$ \\
\hline Presence of Kayser-Fleicher ring & $12(34.29 \%)$ \\
\hline ALT
\end{tabular}

ALT:alanine transaminase; AST: aspartate transaminase; ALP: alkaline phosphatase; IU: international unit; SD: standard deviation

\section{Mutational Analysis:-}

A total of 70 alleles belong to 35 WD patients and other 20 alleles belong to healthy individuals have been examined for mutations in the exon 11 and exon 13 of ATP7B gene. Mutations were detected in 19 WD patients out of 35 $(54.29 \%)$. Seven different mutations have been recorded, three of which are single nucleotide polymorphisms (table 2).

Table 2:- Characteristics of the mutations and the affected domain of $A T P 7 B$ gene in WD patients

\begin{tabular}{|l|l|l|c|l|l|}
\hline Mutation & $\begin{array}{l}\text { Nucleotide } \\
\text { change }\end{array}$ & Type & Exon & $\begin{array}{l}\text { No of alleles } \\
(\%)\end{array}$ & $\begin{array}{l}\text { Affected protein } \\
\text { domain }\end{array}$ \\
\hline Lys832Arg $^{*}$ & c.2495C>T & Missense & 10 & $16(22.86 \%)$ & TM4 \\
\hline Pro840Leu & c.2519C>T & Missense & 10 & $18(25.71 \%)$ & TD \\
\hline Thr991Thr & c.2973A>G & Silent & 13 & $3(4.29 \%)$ & $\mathrm{Ch} / \mathrm{TM} 6$ \\
\hline Ala1003Val & c.3008C>T & Missense & 13 & $6(8.57 \%)$ & $\mathrm{TM} 6 / \mathrm{Ph}$ \\
\hline Lys1010Arg & c.3029A>G & Missense & 13 & $8(11.42 \%)$ & $\mathrm{TM} 6 / \mathrm{Ph}$ \\
\hline c.2977-2978insA & Insertion A & Frameshift & 10 & $17(24.29 \%)$ & $\mathrm{Ch} / \mathrm{TM6}$ \\
\hline c.2457delA & DeletionA & Frameshift & 10 & $19(27.14 \%)$ & $\mathrm{TM} / \mathrm{TD}$ \\
\hline
\end{tabular}

: single nucleotide polymorphism, TM: transmembranous domain, TD: transduction domain, Ch: channel, Ph: phosphorylation

The variant Lys832Arg (rs1061472) appeared in three genotypes; CC, CT and TT (figure 1). Six patients had the heterozygous form and two patients had the homozygous form of the SNP. On the other hand three individuals from control group were carriers for homozygous mutant allele while two of them were carrying herezygous mutant allele of this SNP. 


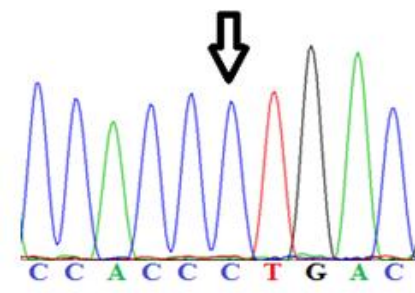

(A)

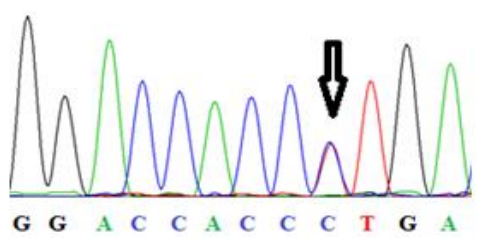

(B)

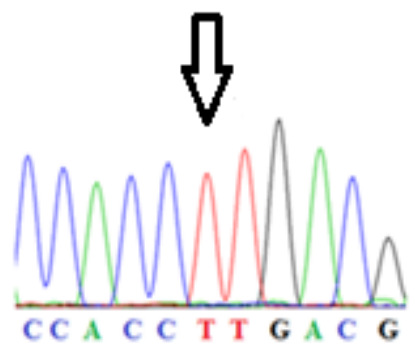

(c)

Figure 1:- Different genotypes of the variant Lys832Arg (rs1061472), forward strand. A:homozygous mutant type allele (CC), B: heterozygous allele (CT), C: homozygous wild type allele (TT).

Similarly, the SNP rs768671894 (c.2519C >T, p.Pro840Leu) appeared in three genotypes (GG, AG and AA, figure 2). Eight WD patients had homozygous mutant genotype and two patients had heterozygote, while all healthy control group had homozygous wild type genotype.

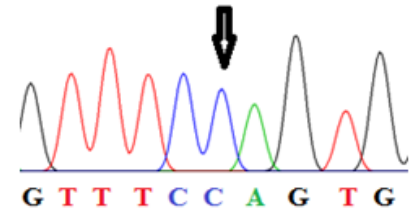

(A)

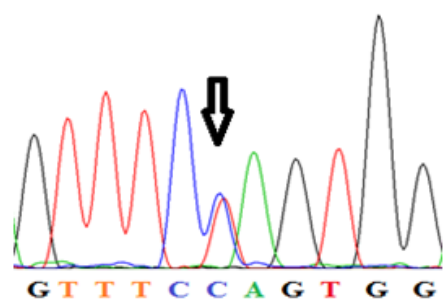

(B)

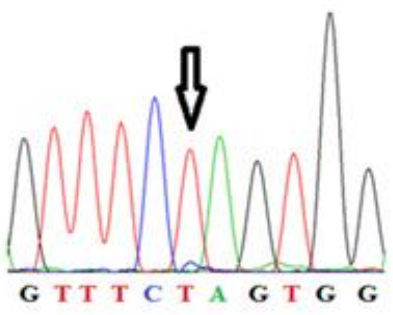

(C)

Figure 2:- Different genotypes of the variant Pro840Leu (rs768671894), reverse strand A:homozygous wild type allele (CC), B: heterozygous allele (CT), C: homozygous mutant type allele (TT).

The synonymous single nucleotide polymorphism rs1801246 (c.2973G>A (p.Thr991Thr) appeared in mutant homozygous form in only 3 patients, with the other patients and controls carrying wild type homozygous genotype (figure 3).

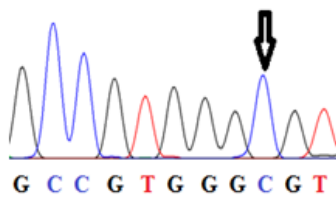

(A)

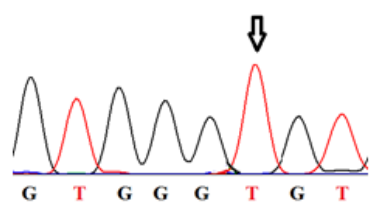

(B)

Figure 3:- Different genotypes of the variant Thr991Thr (rs1801246), forward strand A:homozygous wild type genotype (CC), B: homozygous mutant genotype (TT).

The mutation c.3008C > T (Ala1003Val) affected three patients all of whom were homozygous (figure 4).

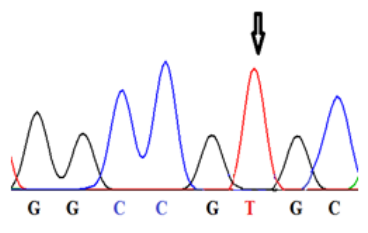

3 (A)

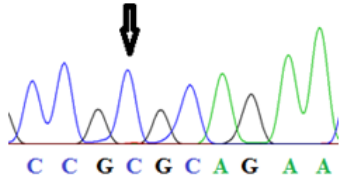

(B)

Figure 4:- c.3008 C>T (Ala1003Val), forward strand A:homozygous mutant (TT), B: homozygous wild type(CC). 
The mutation c.3029A>G (Lys1010Arg) appeared in four WD patients all of whom were homozygote (figure 5).

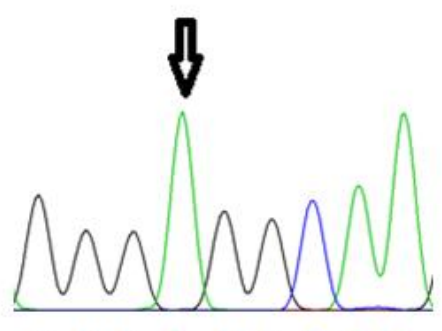

G G G A G G C A A

(A)

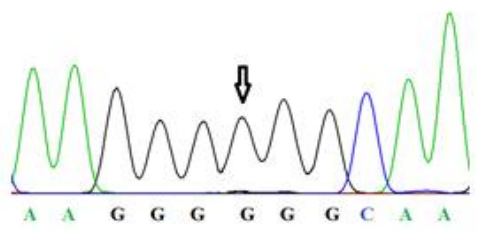

(B)

Figure 5:- c.3029A $>$ G, forward strand A:homozygous mutant (TT), B: homozygous wild type(CC).

This study involved two novel mutations. The first one is c.2977-2978insA (figure 6). This frame-shift mutation affected 17 patients in heterozygous pattern, while 2 healthy controls also affect in the same manner. Thus, it seems that this insertion has neither diagnostic no etiological effect on the disease.

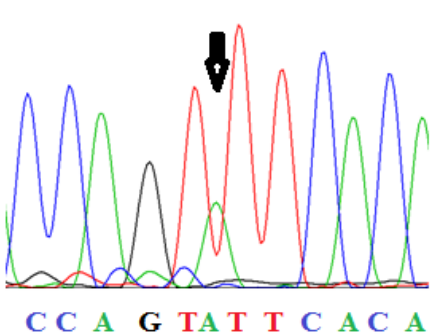

(A)

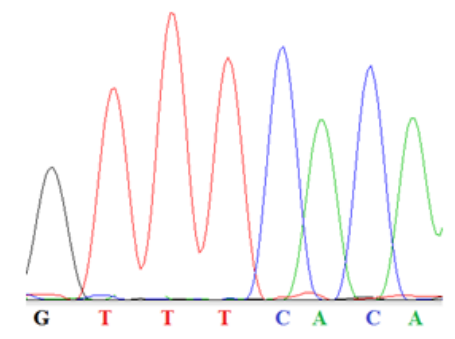

(B)

Figure 6:- (A): c.2977-2978insA, (B): normal sequence

The other mutation is c.2456A Del (figure 7). This is the most prevalent mutation affecting 19 WD patients and absent from healthy control.

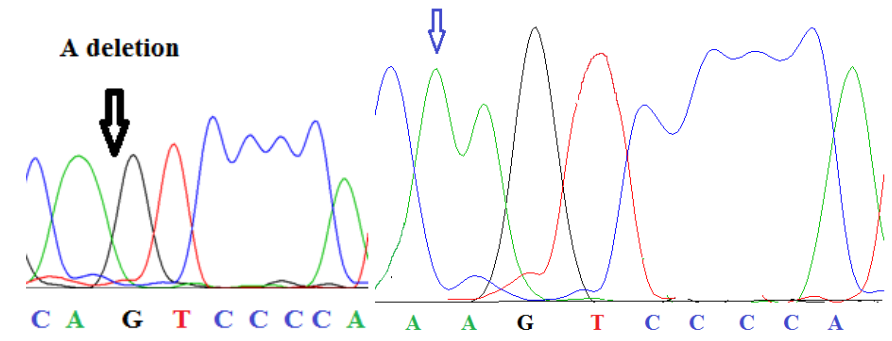

(A) (B)

Figure 7:- (A): c.2457delA, (B): normal sequence

\section{Discussion:-}

This study aimed to analyze the mutational profile of exon 10 and exon 13 of ATP7B gene in patients with WD. Seven different mutations have been recorded, three of which are single nucleotide polymorphisms. The polymorphismLys832Arg (c.2495C $>\mathrm{T})$ is one of the most recorded variants in mutational analysis of ATP7B gene. For example, Gupta et al. (2007) were considered it among the four most common variants in Indian patients with WD which were c.1216 TCT_GCT/p.Ser406Ala, c.2495 AAG_AGG/ p.Lys832Arg, c.2855 AGA_AAA/p.Arg952Lys, and c.1544-53A_C, and recommend to use these for molecular diagnosis of the disease. In Iran, Zali et al. (2011) recorded 0.31 frequency of this variant among Iranian patients, however, the authors found 
it more prevalent among control group (0.38). Recently, Papur et al. (2015) recorded this SNP among five other polymorphisms in the entire ATP7B gene of Turkish patients with WD. More recently, in China, Dong et al. (2016) conducted the largest study in this regard. They sequenced the ATP7B gene from 632 DW patients and 503 unrelated phenotypically normal individuals. Among the 161 variants recorded in this study, the homozygous mutant genotype of the SNP rs1061472 was reported in 105 WD patients, while the heterozygous genotype in 59 patients.

Regardless of the prevalence of this polymorphism, it appears to have no causal relationship with the disease because similar or even higher prevalence of the mutant allele were recorded among normal population.

The SNP rs768671894 (c.2519C>T, p.Pro840Leu) was recorded only in WD patients which reflects its importance in the etiology and diagnosis of the disease. Among the available researches, very rare reports which pointed out this variant in the analysis of mutations of ATP7B gene in WD patients. This variant was previously reported by Zali et al (2011) in one Iranian patients with WD. Interestingly, Dong et al (2016) did not find this variant neither in WD patients nor in healthy control of Chinese population.

The SNP lies in the transduction domain which converts energy from ATP hydrolysis to cation transporter. It involves the substitution of proline with leucine. Poline is a very unusual amino acid, in that the side chain localizes back on to the backbone amide position. The distinctive cyclic structure of proline's side chain gives proline an exceptional conformational rigidity compared to other amino acids. It is because of this property of proline and the position of the variant, it can be postulated that the substitution of proline with leucine reduce the ability of protein to bind ATP and eventual reduction in the ATP7B protein transport the copper outside the manufacturer's hepatocyte. This assumption was previously proposed by Raj and Stanley (1995) who demonstrated that the ATP binding activity of the protein was significantly weakened by the absence of proline in its ATP-binding domain.

The c.2973G>A (Thr991Thr) variant was recorded by Khan et al. (2012) in four out of 90 Indian patients with WD. Approximately, similar frequency was recorded by Dong et al. (2016) in Chinese patients. Other researchers in as many as 10 countries also reported this SNP (Gupta et al., 2007).

As different forms of this variant do not cause amino acid substitution and the minor frequency allele has very low frequency, it does not likely have neither etiological nor diagnostic importance among Iraqi patients.

Thec.3008 C>T (Ala1003Val)mutation is among the most prevalent variant associated with WD worldwide. It was previously found by Papur et al. (2013) in 1\% of Turkish patients and by Santhosh et al. (2006) in Chinese patients. Recently Dong et al. (2016) found this mutation in 51 out of 632 Chinese patients with WD. The mutation lies within the ATP phosphorylation domain of ATP7B protein. The amino acid substitution implied in this mutation was predicted to be very deleterious for the protein function. Alanine is probably the dullest amino acid. It is not particularly hydrophobic and is nonpolar. The alanine side chain is very non-reactive, and is thus rarely directly involved in protein function, but it can play a role in substrate recognition or specificity, particularly in interactions with other non-reactive atoms such as carbon (Matthew et al., 2003).

On the other hand, valine prefers to be buried in protein hydrophobic cores. Whereas most amino acids contain only one non-hydrogen substituent attached to their $\mathrm{C} \beta$ carbon, valine contains two. This means that there is a lot more bulkiness near to the protein backbone which influences the ability of the main chain of this amino acid for adoption in to diferent. Perhaps the most pronounced effect of this is that it is more difficult for this amino acid to adopt an $\alpha$-helical conformation. Due to these differences between the two amino acids, it is reasonable to suppose some deterioration effect of valine on the activity of ATP7B protein in the phosphorylation of ATP and then the deposition of copper inside the hepatocytes.

The mutationLys1010Arg (c.3029A>G) was previously reported by Santhosh et al. (2006) and Gupta et al. (2007) in South Indian hepatic patients, while Dong et al found it in $16 \%$ of the Chinese patients with WD.

Arginine and lysine are positively charged basic amino acids under physiological conditions (Yokota et al., 2006). The two amino acids play important roles in protein stability by forming ionic interactions and hydrogen bonds in the proteins as well as by interacting with water molecules (Strickler et al., 2006). Although they both function as basic residues, the arginine residue provides the protein structure with more stability than lysine owing to its 
geometric structure. The guanidinium group in arginine allows interactions in three possible directions through its three asymmetrical nitrogen atoms, whereas only one direction of interaction is allowed by the basic functional group of lysine (Donald et al., 2011). This enables arginine to form a large number of electrostatic interactions, such as saltbridges and hydrogen bonds compared to lysine, which presumably results in stronger interactions than the interactions generated by lysine (Chan et al., 2011). In addition to the geometric effect, the ionic interactions formed by arginine can be more stable than those of lysines, particularly under alkaline pH (Turunen et al., 2002). Sokalingam et al. (2012) showed that the mutagenesis of lysine to arginine can induce changes in the electrostatic interactions in an additional manner, which might be a factor in enhancing the stability; and such mutagenesis can affect the protein folding unfavorably. This may explain partially the defect of ATP7B protein function in the phosphorylation of ATPase. Thus, this mutation involves a deleterious effect on the transduction domain which converts energy from ATP hydrolysis to cation transporter, and therefore it may be considered as one of the important mutation associated with the disease.

This study involved two novel mutations; c.2977-2978insA and is c.2456A del. The first one seems to have no diagnostic or etiological effect on the disease as it was seen in two controls. On the other hand, c.2456A del is the most prevalent mutation affecting 19 WD patients and was absent from healthy control. It involves a deleterious effect on the transduction domain which converts energy from ATP hydrolysis to cation transporter, and therefore it may be considered as one of the important mutation associated with the disease. Furthermore, it may be used in combination with other prevalent mutation in order to establish a molecular test for diagnosis of the disease. However, such test needs further investigations to determine which mutations are the most prevalent.

Collectively, these data indicate that both c. 2519C > T polymorphism and the frame-shift mutation c.2977-2978insA are candidate mutations that could be exploited in the development of molecular diagnosis of WD. However, further studies are required to find out the most prevalent mutations in other exons.

\section{Acknowledgements:-}

We thank Dr. Halla/ Al-Kadimiya Hospital for help in collection of patient samples and Dr. Qasim Sharhan for critical comments and technical assistance.

\section{Competing Interests:-}

The authors have declared that no competing interest exists.

\section{Reference:-}

1. Al-Mayah QS, Arif HS, Shamran HA et al. (2016). Mutational profile of exon 14 and exon 21 of ATP7B gene in patients with Wilson disease. Unpubplished data

2. Chan C-H, Yu T-H, Wong K-B (2011). Stabilizing Salt-Bridge Enhances protein

3. Chen, C. et al., (2015) . Currently Clinical Views on Genetics of Wilson's Disease. Chinese medical journal, 128(13), pp.1826-1830.

4. Cocoş, R., et al., (2014). Genotype-Phenotype Correlations in a Mountain Population Community with High Prevalence of Wilson's Disease: Genetic and Cinical Homogeneity. PloS one, 9(6), p.e98520.

5. Donald JE, Kulp DW, DeGrado WF (2011). Salt bridges: Geometrically specific, designable interactions. Proteins: StructFunctBioinform,79: 898-915.

6. Dong Y, Ni, W, Chen, W.J, et al (2016).Spectrum and Classification of ATP7BVariants in a Large Cohort of Chinese Patients with Wilson's Disease Guides Genetic Diagnosis. Theranostics ; 6(5):638-649.

7. Dong, Q.Y. \& Wu, Z.-Y. (2012). Advance in the pathogenesis and treatment of Wilson disease. Translational neurodegeneration, 1(1), pp.23-31. e21624.

8. European Association for the Study of liver(2012).http://www.medscape.com.

9. Ferenci, P.,(2006). Regional distribution of mutations of the $A T P 7 B$ gene in patients with Wilson disease: impact on genetic testing. Human genetics, 120(2), pp.151-159.

10. Gupta, A. et al .(2007). Molecular Diagnosis of Wilson Disease Using Prevalent Mutations and Informative SingleNucleotide Polymorphism Markers. ClinicalChemistry. 53: 1601-1608

11. Javed, M.A. et al.,(2008). Neurological and neuropsychiatric spectrum of wilson's disease in local population ,Biomedica:24. 
12. Khan et al (2012). Mutation Profile in Wilson's Disease from North Indian Patients . Int J Hum Genet, 12(2): 125-132.

13. Kodama, H., Fujisawa, C. \& Bhadhprasit, W.,(2012). Inherited Copper Transport Disorders: Biochemical Mechanisms, Diagnosis and Treatment. Current Drug Metabolism, 13(3), pp.237-250.

14. Li, X.-H. et al.,(2011). Clinical and molecular characterization of Wilson's disease in China: identification of 14 novel mutations. BMC medical genetics, 12, p.6.

15. Mathur, M. et al., (2015). Development of low-density oligonucleotide microarrays for detecting mutations causing wilson' s disease. Indian J Med Res. pp.175-186.

16. Matthew, J., Betts and Robert, B., Russell,(2003).Amino Acid Properties and Consequences of Substitutions.Bioinformatics for Geneticists,pp. 291 - 316.

17. Papur, O., Ş., A., A, Sezin ,Terzioğlu ,O. (2015) .Clinical and genetic analysis of pediatric patients with Wilson disease. Department of Medical Biology and Genetics, Dokuz Eylül University Faculty of Medicine, İzmir, Turkey 2 Department of Pediatric Gastroenterology, Hepatology and Nutrition, The Ministry of Health Tepecik Teaching and Research Hospital, İzmir, Turkey, 26: 397-403 .

18. Raj, K., and Stanley ,M.,A.,(1995) .The ATP-binding and ATPase activities of human papillomavirus type 16 $\mathrm{E} 1$ are significantly weakened by the absence of prolines in its ATP-binding domain. J Gen Virol, 76 ( Pt 12):2949-56.

19. Santhosh,S.,Shaji, RV.,Eapen,CE.,et al.,(2006). ATP7B mutations in families in a predominantly Southern Indian cohort of Wilson's disease patients. Indian J Gastroenterol, 25:277-282 .

20. Schilsky M. L.,(2007) • Diagnosis and Long-Term Management of Wilson Disease.Gastroenterology \&Hepatology, 3(1):27-29.

21. Sokalingam,S., Raghunathan,G., Soundrarajan, N.,(2012).A Study on the Effect of Surface Lysine to Arginine Mutagenesis on Protein Stability and Structure Using Green Fluorescent Protein .Department of Chemical Engineering, Pusan National University, Busan, South Korea , Available at: http://journals.plos.org/plosone/article/asset?id=10.1371\%2Fjournal.pone.0040410.PDF

22. Strickler, SS., Gribenko AV, GribenkoAV,et al., (2006). Protein Stability and Surface Electrostatics: A Charged Relationship. Biochemistry 45: 2761-2766.Thermostabilityby Reducing the Heat Capacity Change of Unfolding . PLoSOne6 :

23. Turunen O, vuorio M, Fenel F, Leisola M (2002). Engineering of multiple arginines into the Ser/Thr surface of Trichodermaressei endo-1,4-b-xylanase IIincreases the thermotolerance and shifts the $\mathrm{pH}$ optimum towards alkaline pH.Protein Eng , 15: 141-145.

24. Wu, F. et al., (2015). Wilson's Disease: A Comprehensive Review of the Molecular Mechanisms. International Journal of Molecular Sciences, 16(3), pp.6419-6431. Available at: http://www.mdpi.com/14220067/16/3/6419/.

25. Yokota K, Satou K, Ohki S-y., (2006).Comparative analysis of protein thermostability: Differences in amino acid content and substitution at the surfaces and in the core regions of thermophilic and mesophilic proteins. Science and Technology of Advanced Materials 7: 255-262.

26. Zali, N. et al., (2011). Prevalence of ATP7B Gene Mutations in Iranian Patients With Wilson Disease. Hepatitis monthly, 11(11), pp.890-4. 